\title{
Eliminating Contamination Issues in Manufacturing of Nutritional Products Using Six Sigma Approach
}

\author{
Maxim Krotov and Sanjay Mathrani \\ School of Engineering and Advanced Technology, Massey University, \\ Auckland, New Zealand \\ E-mail:maxkrotov88@gmail.com; s.mathrani@massey.ac.nz
}

Received 12 August 2017; Accepted 11 October 2017;

Publication 7 November 2017

\begin{abstract}
The industry of nutritional products and premixes does not forgive poor quality. It is crucial manufacturers get product right the first time. However, the quality in this industry is always under threat of contamination issues with out-of-specifications and foreign matter impurity being always on the radar of quality control. Six Sigma approach is renowned for improving quality through eliminating variation. It focuses on critical processes that affect the quality for internal and external customer. This paper aims to describe the journey of improving the quality management through development and application of Six Sigma framework using carefully selected tools, specifically tailored for medium-sized food manufacturing business in Auckland, New Zealand. This facility is experiencing contamination issues in their products due to nonconformance with the standard specifications on ingredient quantity or presence of foreign matter within the end product. Defective products have caused a spike in late deliveries and rejections, spoiling the long-earned reputation amongst loyal customers. A framework is developed that embodies Six Sigma methods Define, Measure, Analyze, Control and Improve for food manufacturing business. Using this framework, the study achieves the optimization in loading, mixing and blending processes as well as product packaging. The results describe the improvement in meeting
\end{abstract}

Journal of Industrial Engineering and Management Science, Vol. 1, 225-240. doi: 10.13052/jiems2446-1822.2017.011

This is an Open Access publication. (c) 2017 the Author(s). All rights reserved. 
manufacturing standards, on-time deliveries and reduction of rework and waste. This research provides direction to other small- medium-sized food manufacturing companies in enhancing quality management through eliminating variation in the production processes by effectively adopting the Six Sigma framework.

Keywords: Six Sigma, DMAIC, Quality management, Contamination, Food manufacturing, Nutritional products, Powder mixing.

\section{Introduction}

Today's customer-driven market is highly intolerant to poor quality, especially in food sector, where requirements are stringent and health related. Powder premixes, such as infant formulae in particular are always under pressure to deliver 'right the first time' products following strict standards and regulations on contamination of certain ingredients. Many manufacturing businesses adopt various quality improvement models for enhancing the quality management throughout all the processes. Numerous studies show the success of Six Sigma in a manufacturing environment in terms of quality, delivery times and customer satisfaction [1-4].

Six Sigma methodology is one of the most popular models for improvement of quality control and management. It was introduced in 1987 at Motorola [5] and gained popularity among manufacturing enterprises of all sizes within different industries as an effective quality improvement toolset. Successful applications of Six Sigma within manufacturing businesses show major reduction of failure through complete focus on performance characteristics, which are critical to customers [6]. The improvements include enhanced product quality for internal and external customer, decreased variation and saved costs [1, 3, 7-9]. The Define, Measure, Analyse, Improve and Control (DMAIC) framework is the cornerstone of Six Sigma philosophy, evolved from Deming's Plan, Do, Check, Act cycle [5].

Despite the number of case studies on successful application of Six Sigma towards quality improvement within manufacturing, little insights are discussed particularly on Six Sigma use in small- and medium sized food manufacturing enterprises. Food industry comprises strict regulations upon variation in quality. Moreover, an adoption of an improvement model requires extra investments and resources, which SMEs usually cannot afford. Thus, they are looking for simplified and optimized frameworks, which will benefit 
the company both short- and long-term. Literature highlights the need for further development of Six Sigma application throughout different industries, including food sector [1, 3, 7-9].

This study investigates the applicability of Six Sigma methodology towards improving quality management in an infant formula manufacturing facility and determine the most suitable framework alongside an appropriate toolkit with a view on the food industry. The core research problem is to evaluate the effect of Six Sigma methods on quality in a food manufacturing facility. The study follows the DMAIC framework and pursues the following objectives:

1. To determine all the possible root-causes of failures, contributing to the poor quality;

2. To study hotspots, identify improvement solutions.

Managers of SMEs in food sector could follow the framework presented with the tools used in this study and implement in their quality improvement projects. Researchers could use this study and results as a good example of successful Six Sigma application in New Zealand food manufacturing facility as a part of future more detailed studies.

\section{Literature Review}

Six Sigma is a quality improvement approach which combines a number of the time-proven tools and techniques, optimized for the use in the modern day's industries [10]. It aims to eliminate failures within business processes through complete focus on performance characteristics, which are critical to customers [6]. The Define, Measure, Analyse, Improve, Control (DMAIC) framework is the cornerstone of Six Sigma philosophy, evolved from Deming's Plan, Do, Check, Act cycle [10]. Six Sigma is a vertical approach in improving quality within business processes, which reflects a systematic and well-structured methodology in problem-solving [11].

Although each case study on Six Sigma is unique, there are general improvements that companies aim to achieve. Successful case studies show reduction of defects and variation of processes as a baseline improvement. It is followed by shorter cycle times, saved production costs, reduction of waste and inventory levels and improved customer satisfaction. As a result, successful companies enjoy growing market share and newly created production capacity $[1,2,4,12,13]$. 
The main framework for Six Sigma implementation is DMAIC. It is a cyclic improvement model which abbreviates for define, measure, analyze, improve, control [5]. Design for six sigma is used in development of a new product/service, where quality is concerned at the very beginning [14]. Therefore, DMAIC would be the first choice for companies who want to improve the quality of the end products and optimize the production, before introducing new products.

Implementations of Six Sigma in food sector face their own challenges perishable products, high standards of quality and variation in supply chain. Studies also highlight similar patterns in improvements as optimization of operations and reduction of cycle times are achieved. Researchers agree, that this is only possible through communication, alignment and commitment within the whole organization. The frameworks used in food sector extensively use statistical tools, as producers gather large amounts of data, but have no analysis tools or protocols $[1,2,4,12,13]$.

\section{Research Methodology}

This research follows the qualitative case study and action research methodology. It focuses on answering questions "why" - why do defects occur; and "how" - how to prevent defects from occurring. Moreover, another underpinning for conducting a case study within this research is that according to Yin [15], researchers follow this methodology when it is not possible to manipulate and control the behavior of the variables/people involved in the study. Action research has been chosen as it focuses on learning by doing [16], that is making improvements and evaluating their importance and further suitability within the context of the study.

The context company - Vitamin Premixes (VPX - pseudonym) had been experiencing major quality issues and were on a lookout for quality management improvement models. VPX is a typical example of a NZ manufacturing SME. The company specializes in dry powder mixing with the main product being infant formulae.

This action research commenced in March 2016. Visits were made to the Vitamin Premixes' factory on a fortnightly basis. In August and September, when main improvements were implemented, factory visits were more frequent, as situation required. Data were collected via ethnography, semi-structured interviews and electronic datasheets.

In the ethnography phase, hours were spent on the shop floor, observing workers perform their daily routine tasks. Packaging and handling processes 
were of special interest during this phase, as there were no related metrics available. The importance of those processes are discussed later.

Semi-structured interviews were held at the site, in manager's offices or meeting rooms, and lasted between 30 to 90 minutes. These were conducted according to Massey University's code of ethics and were subject to the non-disclosure policy of the University and the company. Respondents were selected upon their area of expertise and degree of knowledge in certain fields. This study required specialists from quality assurance (QA), operations and manufacturing departments. Open-ended questions were asked to the immediate employees at the workstations and in the departments of interest, such as handling, mixing and packaging.

Data were also collected from electronic sources - Manufacturing output and Testing submissions spreadsheets were available for analysis in this study. They consisted of all the information about goods produced in terms of timestamps, batch and product numbers, processes involved, testing results, reasons for rejection and target dates.

The theoretical methodology for conduct of the study in Six Sigma approach for quality improvement at a food supplement manufacturing company is presented below. It follows the DMAIC approach. Topical tools are highlighted within every stage of the framework below. Table 1 represents the action plan, that is followed through the study.

\section{1) Define}

The problem of the out-of-spec end products is the starting point. Further insights were required for defining the problem, which were gathered through brainstorming and evaluating the current state. Project charter and Gantt chart $[2,12]$ were used.

\section{2) Measure}

Variation in processes and bottlenecks were identified. Continuous and discrete measures were collected. Quality datasheets were obtained from the company

Table 1 DMAIC Action Plan, adapted from Dora [2]

\begin{tabular}{lllll}
\hline Define & Measure & Analyse & Improve & Control \\
\hline $\begin{array}{l}\text { Problem } \\
\text { definition }\end{array}$ & Data collection & Root-cause analysis & $\begin{array}{l}\text { Design of } \\
\text { Experiment }\end{array}$ & Training \\
\hline $\begin{array}{l}\text { Brain- } \\
\text { storming }\end{array}$ & $\begin{array}{l}\text { Measurement } \\
\text { system analysis }\end{array}$ & $\begin{array}{l}\text { Sources of variation, } \\
\text { significance }\end{array}$ & $\begin{array}{l}\text { Implementation of } \\
\text { improvements }\end{array}$ & $\begin{array}{l}\text { Communicate the } \\
\text { improvements }\end{array}$ \\
\hline $\begin{array}{l}\text { Current } \\
\text { state }\end{array}$ & $\begin{array}{l}\text { Trending } \\
\text { defects }\end{array}$ & CTQ characteristics & Effect assessment & Visual management \\
\hline
\end{tabular}


reports as well as from employees face-to-face. Measurement assessment tree was built for the evaluation [5].

\section{3) Analyse}

Measured data were analyzed using several statistical tools. Pareto charts were used to determine main causes of the issue [5]. Fishbone diagrams helped to qualify all the possible causes for defects and to potentially identify hidden and overlooked ones.

\section{4) Improve}

Improvements were designed, evaluated and proposed using design of experiments [11]. The solution was modelled using statistical tools and Minitab software.

\section{5) Control}

With the improvements made, the main challenge was not to let the change slip. This was achieved through visual management boards and trainings in order to adapt staff to the new standards and changes in processes. The purpose was to turn new processes into a routine.

\section{Case Study}

\subsection{Company Background}

Vitamin Premixes company is committed on delivering top quality, innovative and sustainable products to the customers. However, VPX have been experiencing major quality issues in their production facility. The Auckland branch employs 80 people, operates 5 days per week, $2 \times 8$-hour shifts per day. Their production has been on the decline over the past three years: from 2154 batches produced in 2014 to 1445 - in 2016 (projected), due to which the company has lost a significant amount of its market share. Moreover, VPX have recently lost one of their largest customers due to the poor quality products.

As a result of constantly arising quality issues, a number of customer complaints concerning variation of ingredient contamination and presence of foreign matter have been reported. Apart from spoilt reputation, this has led to a surge in costs and wastage of time. The rejected products could neither be recycled nor reused, but destroyed. Hence, the top management decided 
to implement Six Sigma at the facility to improve quality and recover market share and customer trust.

\subsection{Action Research Implementation}

Action research is carried out following the specified action plan (Table 1) and Six Sigma DMAIC framework.

\section{1) Define phase}

A clear problem statement is essential for the execution of Six Sigma Define stage:

"Persistent variation of ingredients' contamination, falling outside of specified standards, causing consequent issues of rework, late deliveries, unforeseen backorders".

The flow of this study was guided by the quality assurance manager and the project charter was initially created. The initial goal for Six Sigma was to underline the easiest improvements to implement or in other words find and pick "low hanging fruits". Brainstorming sessions were carried out with operations manager, QA officers, plant manager, laboratory technician and head of sales to gain their perspective on possible causes of failure and also identify critical to quality criteria, mainly based on voice of customer. The identified possible causes included inconsistent batch sizes and blender loads, raw material qualification (RMQ), end product wait times between mixing and packaging and overall level of hygiene at the facility.

In order to obtain an insight and overview of the operations, process flowchart and current state map (Figure 1) were developed. High level value stream map assisted in idea generation for improvement possibilities from raw material acquisition to shipping and waste management. Relevant data were collected from workers responsible for workstations and their managers. The current state map indicated the number of quality checks (QC) performed and showed an excessive number of employees involved in some of the processes. Despite multiple QCs, a product was rejected only after external lab test. That showed the ineffective use of time and human resources, which resulted in long cycle times and contributed to late deliveries. Based on high level maps, improvement ideas were collected and assessed against criteria of ease, cost of implementation and level of benefit of the change. Among others, optimizing blender loads, revising QC and RMQ change were considered as critical and accepted for projects. This study mainly covered blender loading processes optimization. 
M. Krotov and S. Mathrani

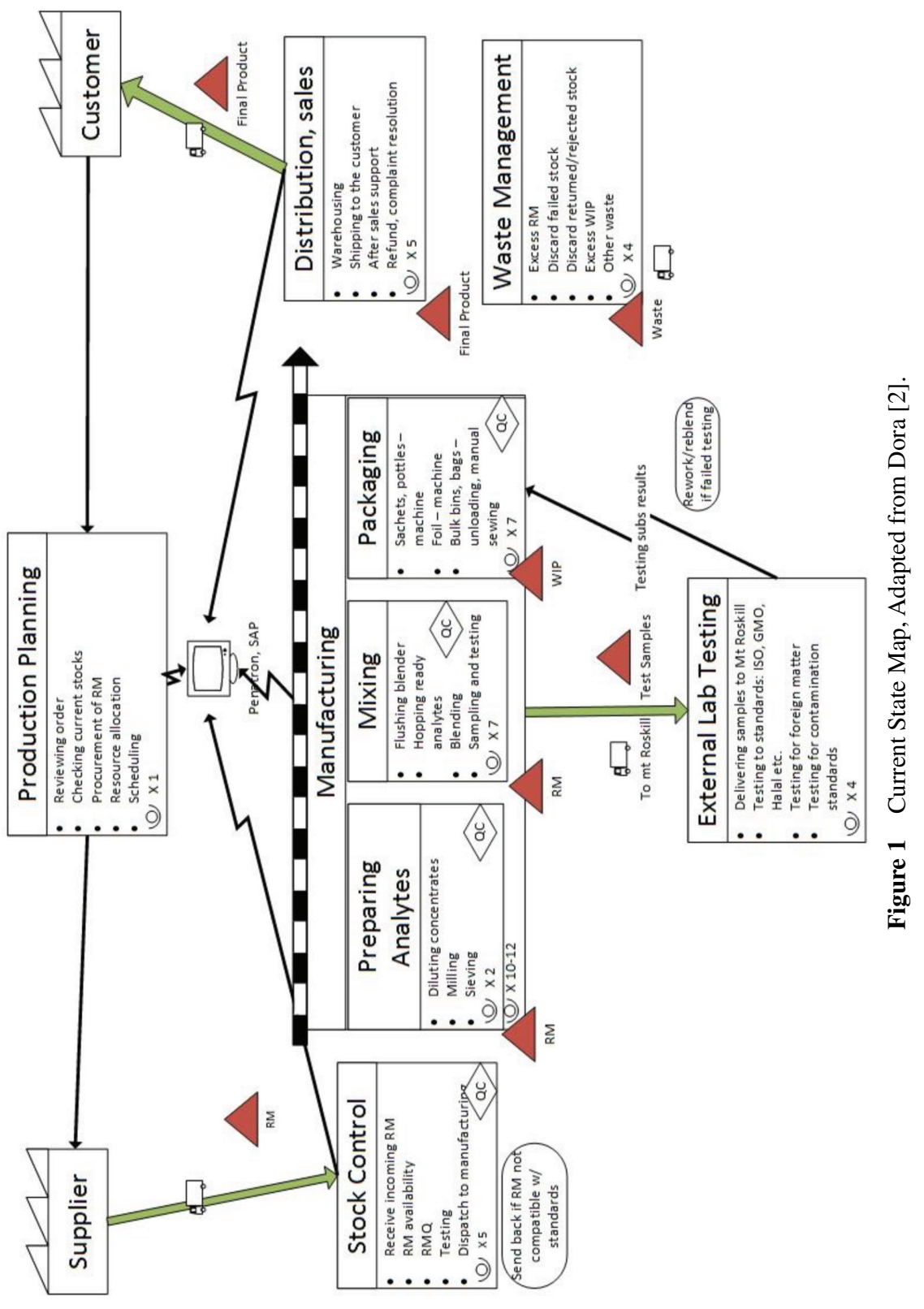




\section{2) Measure phase}

This study used several methods for measuring performance and key characteristics affecting the out-of-specification issue such as:

- Ethnography - spending hours on a shop floor and observing how workers perform routine tasks;

- Semi-structured interviews with heads of quality departments, managers, officers between 30 and 90 minutes long;

- Face-to-face dialogs with employees, starting with "Could you tell me about this process?"

- Data analysis - gathering and analysing of big data from manufacturing output and testing submissions.

Data monitoring included manufacturing output and testing submissions over the year 2016 up until September, as well as data from the past two years. A high-level analysis of testing submissions revealed that failure rates had been on the rise: $10 \%$ of the total output in 2014, 13\% in 2013 and $15 \%$ in 2016. Out of 196 batches, not passing QC, only 20 were reused, 61 were discarded (including 26 containing bacteria) and 76 were dispatched to the customer. Out of dispatched "bad" batches, 27 were rejected and sent back. Late deliveries pushed up from 18\% of total output in 2014 to $47 \%$ in 2016.

Measurement assessment tree showed characteristics that could predict the quality, if those characteristics were present. The main finding was that the facility did not have standard batches and did not measure the wait time of end products before packaging. Waiting times are important, as the end products are highly perishable and may degrade with the time spent in the open area. The absence of standard batches promotes human error and increases the variance of the contamination in the end product, hence negatively affecting quality.

Given company's extensive product range, it was necessary to outline a vital few of trending products and associate ingredients, using Pareto charts. The identified top- 3 trending defective products were identified. The top- 5 trending products made up to $70 \%$ of all the manufacturing output. These three products were chosen for further, more detailed analysis. Within ingredient range, the special interest on Iron, Biotin, Selenium and Vitamin B2 as they caused failure in selected products.

\section{3) Analyse phase}

The objective of this study was to determine the root-causes of failures caused by out-of-specifications and diagnose the most significant processes contributing to the issue. Cross-functional brainstorming sessions identified 
Table 2 Analysis of Variance Results

\begin{tabular}{lll}
\hline Factor & $p$-value & Verdict \\
\hline Blending Tims (BT) & $P=0.369<0.05$ & Insignificant \\
Waiting Time (WT) & $P=0.002<0.05$ & Significant \\
Blender Load (LD) & $P=0.009<0.05$ & Significant \\
Chosen Blender (B) & $P=0.001<0.05$ & Significant \\
Raw material Type (RM) & $P=0.002<0.05$ & Significant \\
Batch Size (SZ) & $P=0.007<0.05$ & Significant \\
Cleaning Process (CL) & $P=0.153<0.05$ & Insignificant \\
\hline
\end{tabular}

and summarized the possible parameters affecting analyte contamination within selected products, all presented in the cause-effect diagram. Key factors and possible sources of variation were determined as blending time (BT), waiting time (between mixing and packaging) (WT), blender load (LD), raw material readiness (RM), chosen blender (B), batch size against standard (SZ), cleaning process before production (CL). The difficulty was that waiting time was never recorded and hence there were no past data. However, once this framework was approved, workers started to record timestamps since May 2016.

Next stage was to shortlist the most significant parameters affecting quality to narrow down further analysis. It was important to assess factors against their data availability, effect on quality and control ability. The analysis was done in two stages - statistical using Minitab to highlight most significant factors and failure mode effect analysis (FMEA) for selected factors from stage one. First stage used data from manufacturing output and testing submissions from 2015 and 2016 for selected products. ANOVA analysis was conducted to determine the most significant contributors to the quality issues. ANOVA has widely been used in the literature within Six Sigma applications [17]. It proved WT, LD, B, RM, SZ to be significant influencers for the contamination of analytes in the final products (Table 2).

Furthermore, FMEA was conducted. Failure modes were modelled for every factor, possible consequences and frequencies were assessed. The goal of FMEA is to prioritise risks, issues which require immediate actions. The criteria for ranking were as follows:

- Occurrence frequency (FR): 1 - failure rarely occurs, 10 - occurs almost daily;

- Severity of failure (SE): 1 - failure causes slight inconvenience to the processes, 10 - failure causes major delays and/or requires substantial costs to repair; 
- Ease of detection (DE): 1 - easy to detect, 10 - hard to detect, no such technology/skill available.

Processes were scored together with the managers or operators of the selected processes, depending on who had better understanding and insightful knowledge. Then, all three criteria were multiplied to get their risk priority number (RPN) and failure modes were ranked accordingly. The most significant have the highest RPN. According to the Table 3, WT and LD turned out to be the most significant factors threatening quality.

\section{4) Improve phase}

Drawing results from ANOVA and FMEA, the study proposed solutions for identified factors. The aim of this stage was to reduce the impact, eliminate the root causes and standardize new operations.

For RMQ, main suggestion was to centralise the filing system, which was currently under development. The improved software would comprise all of the suppliers' information in one place along with the sample packs information. Also, it would have an online system to approve raw materials for manufacturing, eliminating substantial amounts of paperwork. Another proposal was to evaluate the feasibility of ordering ready-to-use RM, rather than concentrates. This would eliminate the current room for error within raw material preparation in the dilution process.

Table 3 Failure Mode and Effect Analysis

\begin{tabular}{|c|c|c|c|c|c|c|c|c|c|}
\hline \# & Factor & Failure & Effect & SE & Cause & FR & Current & $\mathrm{DE}$ & RPN \\
\hline 1 & $\begin{array}{l}\text { Waiting } \\
\text { Time } \\
(\mathrm{WT})\end{array}$ & $\begin{array}{l}\text { Uncovered and } \\
\text { exposed to the } \\
\text { environment } \\
\text { final product } \\
\text { sitting too long } \\
\text { before } \\
\text { packaging }\end{array}$ & $\begin{array}{l}\text { Product } \\
\text { degrades, } \\
\text { prone to } \\
\text { bacteria } \\
\text { and foreign } \\
\text { matter }\end{array}$ & 8 & $\begin{array}{l}\text { Packaging/ } \\
\text { forklift } \\
\text { busy }\end{array}$ & 7 & $\begin{array}{l}\text { Person } \\
\text { from } \\
\text { packaging } \\
\text { comes and } \\
\text { picks up } \\
\text { the bin }\end{array}$ & 7 & 392 \\
\hline$\overline{2}$ & $\begin{array}{l}\text { Blender } \\
\text { Load } \\
\text { (LD) }\end{array}$ & $\begin{array}{l}\text { Blender is too } \\
\text { full or almost } \\
\text { empty }\end{array}$ & $\begin{array}{l}\text { Powder } \\
\text { does not } \\
\text { mix } \\
\text { properly }\end{array}$ & 8 & $\begin{array}{l}\text { Bad } \\
\text { planning }\end{array}$ & 6 & $\begin{array}{l}\text { Planning } \\
\text { aims to the } \\
\text { max } \\
\text { capacity }\end{array}$ & 7 & 336 \\
\hline 3 & $\begin{array}{l}\text { Raw } \\
\text { Material } \\
\text { Type } \\
\text { (RM) }\end{array}$ & $\begin{array}{l}\text { RM are not to } \\
\text { spec from } \\
\text { supplier side or } \\
\text { diluted wrongly } \\
\text { internally }\end{array}$ & $\begin{array}{l}\text { Wrong } \\
\text { amount of } \\
\text { analyte } \\
\text { coming } \\
\text { into premix }\end{array}$ & 7 & $\begin{array}{l}\text { Supplier } \\
\text { or } \\
\text { internal } \\
\text { human } \\
\text { error }\end{array}$ & 4 & $\begin{array}{l}\text { RM are } \\
\text { tested } \\
\text { externally } \\
\text { prior to } \\
\text { mixing }\end{array}$ & 5 & 140 \\
\hline
\end{tabular}


This study has implemented one change in RMQ - all the incoming materials are now tested internally, saving time on travelling between labs as well as utilising human resources more efficiently.

For the issue of WT, this study proposed qualitative improvement. Making employees from the mixing department responsible for covering and moving the bulk bin further to packaging. This way when packaging is busy, products get isolated from the environment, remaining aseptically clean. This has improved the flow of products. Implemented in August - since then, detection of enterobacteriaceae reduced from 37 before August - to only twice.

Improving LD involved statistical tool - design of experiments. It is a trial-error method, aimed to find out close-to-perfect load and standardize the solution. Variables LD, B and SZ were included in the experiment and tuned on an example of an animal mineral premix with higher tolerances to find optimal settings. Once new standards of batch sizes and loads were suggested, confirmation trials were conducted to prove the feasibility of the solution. The "good-practice" capacity sat in the range $45-50 \%$ of the full blender load for large blenders (4427 L) and 55-60\% for smaller ones (1174, $237 \mathrm{~L}$ ), due to their higher rotational velocities. The hopping of ingredients was changed to partial hopping. Only a third of every ingredient was added at one time, making several layers of powder before mixing.

After confirmation, the proposed solution was accepted for a particular product. Within two months there was not a single occurrence of out-of-spec in that product. Overall process capability improved significantly.

All the changes led to the reduction in late deliveries from $47 \%$ to $23 \%$. The forecast for 2017 is to eliminate late deliveries by June, before determining optimum loads for all the major products. Customer complaints stopped coming in, as minor failures are now detected before dispatching.

\section{5) Control phase}

In this stage, the aim was to keep the momentum and sustain the positive change in the long term. The new standards of loading were introduced and communicated within the appropriate departments. Visual management boards were developed and placed in the common areas.

\section{Results and Discussion}

This paper has determined the good practice for implementing Six Sigma for quality improvement with a view on food manufacturing. The combinational use of statistical and visual tools helped the organization to address the change. 
The systematic DMAIC framework has used appropriate statistical tools such as Pareto, ANOVA and DoE. They have well blended with Six Sigma quality tools of Ishikawa diagram, current state maps and measurement assessment trees. The company has experienced improvement in quality and reduction in waste.

The exact failure rates of the total output were identified as rising to $15 \%$ in 2016 along with trends within products (Infant formulae) and ingredients (Biotin, Selenium, Vitamins B2). Inconsistent results of the deliverables created a backlog of work-in-progress and backorders of raw materials, all causing late deliveries to soar to $47 \%$ (2016). Only $10 \%$ of the failed products were reused, others - discarded.

FMEA and ANOVA analyses helped in determining of hotspots - waiting times and blender loads. Waiting times were shortened by reassigning workload away from busier departments, streamlining the flow and optimizing the workload. It resulted in an almost-elimination of enterobacteriaceae within final products. Optimum loads were identified as $45-50 \%$ for the large and 55-60\% for the small blenders. This change was applied to one of the major products and resulted in a significant reduction of out-of-specification occurrences within that product. Altogether, implemented changes have positively influenced facility's performance and flow, proven by halving the late deliveries rate from $47 \%$ to $23 \%$ and aiming to eliminate completely by June 2017.

Six Sigma has proven to be a well-suited quality management and improvement model for the use within medium-sized manufacturing businesses in a New Zealand context. The DMAIC framework described in this study has an appropriate set of tools, applicable for quality management improvement processes in the food manufacturing business.

The results have shown a positive impact on quality, eliminating defects in the major product and significantly reducing late delivery rates, along with increase in overall customer satisfaction. Moreover, the application of Six Sigma helped the facility to create additional manufacturing capacity by streamlining the process flow and utilizing human resources more efficiently. The outcomes of this project are consistent with literature. Companies that adopted Six Sigma DMAIC framework have shown strong decrease in variability and increase in profits [8,9]. In addition, literature has found companies achieve improvements in operational performance $[1,2,18]$. This study is consistent with literature and confirms the main statement covered by authors earlier, i.e., significant improvement of quality and reduction in defective end products $[1-3,8,9,18,19]$. 


\section{Conclusion and Future Research}

This project helped the facility to overcome tough times of poor quality and united the team. Cross-functional communications and jobs done created a collaborative atmosphere within the company. It has given the level of confidence to management and employees, which transitioned into greater value for the customer. More products have reached their customers on time. Additional manufacturing capacity has been created. Six Sigma implementation at the Auckland dry powder mixing facility has shown results, similar to the literature by achieving significant improvement of quality and reduction in defective end products $[1-3,8,9]$.

The confidence in the results should work as a baseline for further research of Six Sigma application in other small-medium manufacturing facilities in New Zealand, including food and dairy sectors. Every phase of DMAIC is a research topic on its own. In future it is possible to look more into control phase, how to sustain the change. Future study could include control over raw material suppliers and controlled environment. Managers may use this framework as a baseline to improve the quality management at medium-sized manufacturing facilities, not just in food sector. Though, every facility is unique and will require personalization of this method.

\section{References}

[1] Gijo, E., and Scaria, J. (2014). Process improvement through Six Sigma with Beta correction: a case study of manufacturing company. Int. J. Adv. Manuf. Technol. 71, 717-730.

[2] Dora, M., et al., (2013). Operational performance and critical success factors of lean manufacturing in European food processing SMEs. Trends Food Sci. Technol. 31, 156-164.

[3] Kumar Sharma, R. and Gopal Sharma, R. (2014). Integrating Six Sigma culture and TPM framework to improve manufacturing performance in SMEs. Qual. Reliab. Eng. Int. 30, 745-765.

[4] Surange, V. (2015). Implementation of Six Sigma to reduce cost of quality: a case study of automobile sector. J. Fail. Anal. Prev. 15, 282-294.

[5] Pande, P., Neuman, R., and Cavanagh, R. (2002). The Six Sigma Way Team Fieldbok. New York, NY: McGraw-Hill.

[6] Snee, R. D. (2010). Lean Six Sigma-getting better all the time. Int. J. Lean Six Sigma 1, 9-29. 
[7] Lobo, S. R., Matawie, K. M., and Samaranayake, P. (2012). Assessment and improvement of quality management capabilities for manufacturing industries in Australia. Total Qual. Manag. Bus. Excell. 23, 103-121.

[8] Prashar, A. (2014). Adoption of Six Sigma DMAIC to reduce cost of poor quality. Int. J. Product. Perform. Manag. 63, 103-126.

[9] Antony, J., Kumar, M., and Madu, C. N. (2005). Six sigma in small-and medium-sized UK manufacturing enterprises: some empirical observations. Int. J. Qual. Reliab. Manag. 22, 860-874.

[10] Pande, P. S., Neuman, R. P., and Cavanagh, R. R. (2000). The Six Sigma Way. New York, NY: McGraw-Hill.

[11] Montgomery, D. C., and Woodall, W. H. (2008). An overview of six sigma. Int. Stat. Rev. 76, 329-346.

[12] Mukhopadhyay, A. (2006). Reduction of yarn packing defects using Six Sigma methods: a case study. Qual. Eng. 18, 189-206.

[13] Desai, D. A., et al. (2015). Curbing variations in packaging process through Six Sigma way in a large-scale food-processing industry. J. Ind. Eng. Int. 11, 119-129.

[14] Edgeman, R., and Dugan, J. (2008). Six Sigma from products to pollution to people. Total Qual. Manag. 19(1/2), 1-9.

[15] Yin, R. (1994). Case Study Research: Design and Methods. Beverly Hills, CA: Sage Publishing.

[16] Gilmore, T., Krantz, J., and Ramirez, R. (1986). Action based modes of inquiry and the host-researcher relationship. Consultation 161, 5.3.

[17] Stamatis, D. H. (2003). Failure Mode and Effect Analysis: FMEA from Theory to Execution. Milwaukee: ASQ Quality Press.

[18] Cheng, C., and Kuan, C. (2012). Research on product reliability improvement by using DMAIC process. Asian J. Qual. 13, 67-76.

[19] Sharma, R., and Sharma, R. (2013). Integrating Six Sigma culture and TPM framework to improve manufacturing performance in SMEs. Qual. Reliab. Eng. Int. 30, 745-765. 


\section{Biographies}

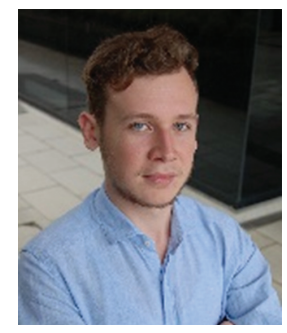

Maxim Krotov received the Bachelor of Engineering with Honours in Product Development from Massey University, New Zealand in 2017. Currently, he works as a Design Engineer at Regal Haulage (an established bulk transport logistics and manufacturing company). He has major knowledge in new product development, process management and manufacturing. He has strong background in mechanical design, particularly in bulk transportation field.

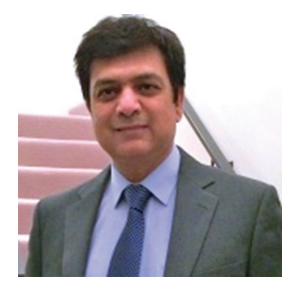

Sanjay Mathrani is a Senior Lecturer in the School of Engineering at Massey University, New Zealand. He holds degrees in Bachelor of Technology in Mechanical Engineering, Masters in Management Science, and a PhD in Information Technology from Massey University. He has a rich industry background with more than twenty years of product development, manufacturing, and global supply chain experience in hi-tech engineering operations. His research interests are in information and knowledge management, product development, and manufacturing operations. He has published several papers in international journals, books, and conferences and is a chartered professional engineer to New Zealand industry. 